\title{
La vigilancia epidemiológica de sarampión y rubéola en el marco del plan de eliminación. Colombia 1995-2009
}
Epidemiological surveillance of measles and German measles (rubella) within the context of the elimination plan: Colombia, 1995-2009

\author{
Lina S. Morón-Duarte ${ }^{1}$ y José O. Castillo-Pabón ${ }^{2}$
}

1 Escuela de Medicina y Ciencias de la Salud, Centro de Investigaciones Ciencias de la Salud, Universidad del Rosario, Bogotá, Colombia. sofismodu@yahoo.com

2 Subdirección de Vigilancia y Control en Salud Pública, Instituto Nacional de Salud, Bogotá, Colombia.

Recibido 7 Abril 2011/Enviado para Modificación 12 Diciembre 2011/Aceptado 28 Diciembre 2011

\section{RESUMEN}

Objetivo Describir el comportamiento de la vigilancia epidemiológica, con el fin de aportar evidencias sobre la interrupción de la circulación endémica de los virus del sarampión y la rubéola en el país.

Métodos Estudio descriptivo retrospectivo de la vigilancia epidemiológica del sarampión y rubéola en Colombia para el período 1995-2009, a través de la consulta de información disponible de notificación en el aplicativo Measles Elimination Surveillance System (MESS), Sistema Nacional de Vigilancia en Salud Pública (SIVIGILA) y el Departamento Nacional de Estadísticas (DANE) para proyecciones poblacionales. La evaluación de la calidad de la vigilancia se hizo mediante los indicadores propuestos para la vigilancia integrada de sarampión y rubéola.

Resultados En el período a estudio se notificaron 28732 casos sospechosos, 66,15 $\%$ de sarampión y $33,8 \%$ de rubéola, mayor número de casos notificados en 2002 (22,4 \%); total de casos confirmados de sarampión entre 1995-2002 ( $n=495)$ y de rubéola ( $n=946$ ) en todo el período. El promedio acumulado de los indicadores fue investigación oportuna con un comportamiento por debajo de $80,5 \%$, notificación semanal por encima de $80 \%$, toma de muestra adecuada en $93,7 \%$, y para recepción de muestra de $65,2 \%$ para $1995-2006$ y de $83,3 \%$ para el periodo $2007-2009$.

Conclusiones De acuerdo con la información aportada por la vigilancia epidemiológica, el país cumple con 4 de los 7 indicadores propuestos para evaluar su calidad. Se evidencia que la circulación endémica de los virus de sarampión y rubéola en Colombia se encuentra interrumpida.

Palabras Clave: Vigilancia epidemiológica, rubéola, sarampión, evaluación (fuente: DeCS, BIREME). 


\section{ABSTRACT}

Objective Describing the behavior of epidemiologic surveillance regarding measles and German measles (rubella) to provide evidence about the interruption of the endemic circulation of these viruses in Colombia.

Methods This was a retrospective descriptive study of epidemiological surveillance for measles and German measles in Colombia from 1995 to 2009 by reviewing available notification information from the measles elimination surveillance system (MESS), the Colombian Public Health Surveillance System (SIVIGILA) and the Colombian Statistics Department (DANE) for population projections. Surveillance quality was evaluated by using the indicators proposed for integrated measles and German measles surveillance.

Results 28,732 suspicious cases were notified during the study period $(66.15 \%$ concerned measles and $33.8 \%$ German measles). The greatest number of notified cases occurred in 2002 (22.4\%); this was detected in the 6-11 month and 2-4 yearold groups. Confirmed measles cases amounted to 495 (1995-2002) and German measles to 946 (for the whole period). The cumulative average for indicators was as follows: timely research had a pattern below $80.5 \%$, weekly notification was above $80 \%$, suitable sampling was $93.7 \%$ on average and sample reception was $65.2 \%$ for $1995-2006$ and $83.3 \%$ for $2007-2009$.

Conclusions According to the information compiled regarding epidemiological surveillance, Colombia complied with 4 of the 7 proposed indicators for quality evaluation; these showed active surveillance having suitable indicator performance regarding laboratory and notification rate. They demonstrated interrupted endemic circulation of measles and German measles in Colombia.

Key Words: Epidemiology surveillance, rubella, measles, evaluation (source: $\mathrm{MeSH}$, $N L M)$.

$\mathrm{E}$ 1 sarampión y la rubéola reúnen los requisitos para ser enfermedades objetivo de eliminación, teniendo en cuenta que su reservorio es exclusivamente humano, los virus apenas sobreviven en el medioambiente; existen técnicas diagnósticas suficientemente sensibles y específicas para detectar la infección, y se dispone de vacunas efectivas y baratas para las que se ha demostrado inmunidad duradera (1); pero aún así, la Organización Mundial de la Salud (OMS) calculó que para el 2008, murieron 164000 personas por causa del sarampión, la mayoría de ellas menores de 5 años, a pesar de la existencia de una vacuna segura, eficaz y del gran impacto en la reducción de la mortalidad por sarampión de las campañas focalizadas de vacunación (2).

Entre 2000 y 2008 se vacunaron aproximadamente 700 millones de niños, de los 9 meses a los 14 años, residentes en países de alto riesgo, y la 
mortalidad por sarampión disminuyó un $90 \%$ en ese período. Los mayores beneficios se obtuvieron en las Regiones del Mediterráneo Oriental y África, donde disminuyó un $90 \%$ y un $89 \%$, respectivamente (3); pero para el 2009 ocurrieron grandes brotes de sarampión en muchos países, la mayoría de ellos ubicados en África, por lo que se teme perder los logros conseguidos en los últimos dieciocho años (4).

En la región de las Américas se consiguió eliminar el sarampión en 2002. Tres regiones de la OMS han fijado objetivos de eliminación, a saber: Mediterráneo Oriental (2010), Europa (2010) y Pacífico Occidental (2012). La región de África estableció un objetivo de pre eliminación en 2008, consistente en reducir la mortalidad por sarampión para 2012 en un $98 \%$ con respecto a las estimaciones de 2000. La región de Asia suroriental es la única que no ha fijado un objetivo de eliminación o pre eliminación: actualmente, esa región está centrando sus esfuerzos en la consecución del objetivo de reducción de la mortalidad por sarampión en todo el mundo $(5,6)$.

El objetivo de la eliminación en las Américas se alcanzó gracias a la aplicación exitosa de la estrategia de vacunación contra el sarampión y la rubéola, que incluye el mejoramiento de los servicios de inmunización sistemática y la realización de actividades suplementarias de inmunización. En consecuencia, todos los países de la Región proporcionan a sus poblaciones por lo menos dos dosis de vacunas contra los virus de sarampión y rubéola. La alta cobertura de vacunación alcanzada mediante la inmunización sistemática y las actividades suplementarias de inmunización han reforzado las probabilidades de eliminación del sarampión, y han acercado a la Región al logro del objetivo regional de eliminación de la rubéola para 2010 . La eficaz aplicación de la estrategia de vacunación contra el sarampión y la rubéola ha dado lugar a un número históricamente bajo de casos de sarampión notificados, que varió entre 85 y 237 casos anuales en el periodo 2003-2008 a raíz de la entrada de virus importados. Dado que el sarampión sigue siendo endémico en otras regiones de mundo, existe el riesgo que se produzcan importaciones del virus, por lo que se debe mantener la estrategia de eliminación hasta tanto todas las regiones hayan eliminado el sarampión (5).

En el 2003, el Consejo Directivo Regional de la OMS, considerando, entre otros, los grandes progresos y la experiencia adquirida por los Estados Miembros en el control acelerado de la rubéola y las iniciativas de prevención del síndrome de rubéola congénita (SRC), estableció la meta de "Eliminación 
de la rubéola y Síndrome de rubéola congénita" en la región para el 2010 (7) y en 2007, se solicitó el inicio del proceso de documentación y verificación de la interrupción de la transmisión endémica del virus del sarampión y la rubéola en las Américas (8). Los países de la región han mostrado un progreso notable en lo que respecta a la interrupción eficaz de la transmisión endémica del virus de la rubéola, a través de la aplicación de estrategias de vacunación, logrando una cobertura del $95 \%$ o más para el 2006 y contribuir a consolidar la eliminación del sarampión (9).

En Colombia, el impacto de la disminución del sarampión se inició tras la inclusión de la vacuna antisarampionosa en 1973 (10). A partir de 1993, el país puso en marcha la iniciativa de eliminación del sarampión iniciando la fase de ataque con la campaña de vacunación "Puesta al Día", cuando se vacunaron cerca de once millones de niños entre nueve meses y catorce años de edad, con un cumplimiento del $97 \%$. En 1995 se realizó la primera campaña de seguimiento, en la que se vacunaron más de 2 millones de niños de 1 a 3 años, con un cumplimiento del $97 \%$ y se inició la aplicación de la vacuna triple viral (contra sarampión, parotiditis y rubéola) al cumplir los 12 meses de edad, esquema que sigue vigente. En 1997 se comenzó a administrar una dosis de refuerzo a los 10 años de edad, con el objeto de avanzar en la eliminación de la rubéola congénita. La segunda campaña de seguimiento se inició en abril de 1999, con el 88,6 \% de cumplimiento (11, 12); en 2002 se realizó la tercera campaña de seguimiento con una cobertura del 94,5 \% (13) y la cuarta campaña de seguimiento en el 2006.

En 2010, los Estados Miembros de la OMS aprobaron una serie de metas provisionales para 2015 hacia la futura erradicación mundial del sarampión. Aunque se han realizado esfuerzos y se han obtenido progresos en la lucha contra el sarampión, se destacan también los retos que hay que superar para alcanzar las metas de 2015; entre ellos figuran los conflictos entre prioridades de salud pública, la fragilidad de los sistemas de vacunación, el mantenimiento de una alta cobertura de vacunación sistemática, soluciones para el déficit de financiación, la vacunación de las poblaciones de difícil acceso y la necesidad de interrumpir el creciente número de brotes de sarampión detectados sobre todo en zonas fronterizas (14). El logro de los objetivos intermedios para 2015 en relación con el sarampión los cuales son conseguir que las coberturas de vacunación del sarampión sean al menos del $90 \%$ a nivel nacional y del $80 \%$ en el nivel local; reducir los casos de sarampión a menos de 5 casos por millón de habitantes y reducir la mortalidad en un $95 \%$ comparándola 
con la mortalidad en el año 2000; son aspectos decisivos si se desea reducir la mortalidad infantil en cumplimiento de los Objetivos de Desarrollo del Milenio (ODM) (15).

En el país se han presentado brotes de sarampión de gran magnitud como el ocurrido en 1993, con 5000 casos, 48 muertes y una tasa de incidencia de 28 por 100000 habitantes. Durante los últimos 10 años la notificación de casos sospechosos al sistema de vigilancia ha presentado un promedio de 2 132 casos, con un rango de 625 en 1997 a 6311 en 2002. En el periodo 1996 - 2001 el número de casos confirmados por laboratorio fue inferior a 10 casos por año, en el 2002 se presentó la última epidemia de sarampión en el país con la confirmación de 139 casos (16).

A partir del 2000, Colombia, inició la integración de la vigilancia de rubéola a la vigilancia ya implementada del sarampión; y, para el 2005 comenzó a efectuar la vigilancia de síndrome de rubéola congénita a través del Sistema de Vigilancia en Salud Pública (17), así como, la fase de eliminación de rubéola y síndrome de rubéola congénita con la Jornada Nacional de Vacunación con sarampión y rubéola dirigida al grupo de 15 a 39 años de edad (18).

Dados estos antecedentes, el objetivo principal de este trabajo es describir los avances del país en la eliminación del sarampión y la rubéola en el componente de vigilancia epidemiológica (19), durante el periodo 19952009, con el fin de aportar evidencias sobre la interrupción de la circulación endémica de los virus del sarampión y la rubéola en el país, además de determinar la calidad de su vigilancia epidemiológica.

\section{MATERIALES Y MÉTODOS}

Estudio descriptivo retrospectivo transversal $(20,21)$ de la vigilancia epidemiológica del sarampión y rubéola en Colombia entre 1995 y 2009. Se consultó la información disponible con el fin de aportar evidencias sobre la interrupción de la circulación endémica de los virus del sarampión y la rubéola en el país a través de la evaluación de indicadores de calidad de la vigilancia epidemiológica.

La fuente principal para dicho estudio fue el aplicativo Measles Elimination Surveillance System (MESS) de la OPS (19), teniendo en cuenta la estabilidad 
y homogeneidad que se ha brindado a partir de dicho sistema de información en la captura y análisis de los datos con respecto a los casos sospechosos tanto de sarampión como de rubéola en el periodo de análisis. La información nacional de dicha fuente es administrada por el Instituto Nacional de Salud, quien actualmente realiza la recepción y consolidación de la información del $25 \%$ (9/36) de las entidades que tienen instalado el software (Antioquía, Atlántico, Bogotá, Cundinamarca, Nariño, Norte de Santander, Sucre, Santander, Risaralda); para el $78 \%$ restante de las entidades territoriales el ingreso al MESS de los casos sospechosos de sarampión y rubéola se realiza desde el nivel central. Adicionalmente fueron consultadas otras fuentes de información como las suministradas por el Sistema Nacional de Vigilancia en Salud Pública (Sivigila) (17), los Laboratorios de Salud Pública Departamentales (LSPD), el Laboratorio Nacional de Referencia (virología INS); y el Departamento Nacional de Estadísticas (DANE), donde se consultaron las proyecciones poblacionales.

En virtud de los cambios sufridos en el sistema de vigilancia epidemiológica y en las actividades de control y eliminación del sarampión y la rubéola en el país, se consideró que la información disponible configuraba una serie interrumpida en la captación de los datos, debido a que en el período analizado se efectuaron intervenciones en la vigilancia y en el programa de vacunación que han modificado de alguna forma los procedimientos de captación de datos: a. Modificaciones en la definición de casos y en los indicadores de vigilancia contenidos en el protocolo de vigilancia respondiendo a las necesidades y criterios nacionales e internacionales; $b$. Inicio de vacuna universal con dos dosis de vacuna de SRP aplicada al año y a los 10 años de edad en 1997; c. Inicio de la vigilancia integrada del sarampión y la rubéola en el 2000; y, d. Campaña de vacunación con la vacuna SR a hombres y mujeres de 14 a 39 años en 2005-2006.

Se tuvieron en cuenta las definiciones de eliminación planteadas por la OPS para sarampión: interrupción de la transmisión endémica del virus del sarampión en todos los países de las Américas por un periodo igual o superior a los 12 meses, en presencia de una vigilancia de alta calidad; $y$, para rubéola: interrupción de la transmisión endémica del virus de la rubéola en todos los países de las Américas por un período igual o superior a los 12 meses sin la ocurrencia de casos de SRC asociados con la transmisión endémica, en presencia de una vigilancia de alta calidad (8). 
Para el análisis de los datos se calculó la frecuencia absoluta anual para: a. Casos sospechosos de sarampión y rubéola; b. Casos sospechosos por grupos de edad; c. Frecuencia y proporción de casos según diagnóstico de clasificación final; d. Incidencia de sarampión y rubéola por 100000 habitantes; y, e. Indicadores de la vigilancia integrada de sarampión y rubéola (Tabla 1).

\section{RESULTADOS}

En el periodo de 1995 a 2009 se notificaron en total 28732 casos sospechosos, $66,15 \%(\mathrm{n}=19006)$ de sarampión y 33,8 \% (n=9 706) de rubéola. En 2002 y 2005 se notificó el mayor número de casos, con $22,4 \%$ ( $\mathrm{n}=6$ 424) y 9,1 $\%(n=2618)$ casos respectivamente (Tabla 2). Los grupos de edad más frecuentes en la captación de casos sospechosos tanto de sarampión como para rubéola fueron los de 6 a 11 meses de edad y de 2 a 4 años.

Tabla 1. Indicadores de la vigilancia integrada de sarampión y rubéola

\begin{tabular}{lc}
\hline \multicolumn{1}{c}{ Indicador } & Meta de cumplimento \% \\
\hline $\begin{array}{l}\text { Proporción de Unidades Primarias Generadoras de Datos } \\
\text { (UPGD) que notifican semanalmente }\end{array}$ & 80 \\
\hline $\begin{array}{l}\text { Proporción de casos con investigación oportuna (48 horas) } \\
\text { Proporción de casos con muestra de suero recolectada en }\end{array}$ & 80 \\
los primeros 30 días luego de iniciada la erupción & 80 \\
$\begin{array}{l}\text { Proporción de muestras de suero recibidas en el laboratorio } \\
\text { en los primeros cinco días luego de su recolección }\end{array}$ & 80 \\
$\begin{array}{l}\text { Proporción de muestras de suero procesadas en el laborato- } \\
\text { rio en los primeros cuatro días luego de su recepción }\end{array}$ & 80 \\
\hline $\begin{array}{l}\text { Tasa de notificación de casos sospechosos de sarampión } \\
\text { y rubéola }\end{array}$ & $\begin{array}{c}\text { Tasa anual de notificación } \geq 2 \\
\text { por } 100 \text { 000 habitantes100 } 000\end{array}$ \\
\hline
\end{tabular}

Durante los años 1995 a 2002 se confirmaron en total 495 casos de sarampión; en el periodo de 2003 a 2009 no se han confirmado casos de esta enfermedad en el país. Para rubéola se han confirmado un total de 946 casos durante todo el periodo en estudio, con una marcada disminución en la frecuencia de casos entre el 2005 y 2006 pasando de 84 casos a 6 casos confirmados. En los últimos 3 años se han confirmado 12 casos de rubéola de los cuales 10 fueron por clínica (error en la vigilancia) y 2 por laboratorio (Tabla 3). 
Tabla 2. Casos sospechosos de sarampión y rubéola notificados entre 1995 y 2009, Colombia

\begin{tabular}{ccccc}
\hline Año & $\begin{array}{c}\text { Sospechoso } \\
\text { sarampión }\end{array}$ & $\begin{array}{c}\text { Sospechoso } \\
\text { rubéola }\end{array}$ & Desconocido & Total \\
\hline 1995 & 264 & 0 & 0 & 264 \\
1996 & 1032 & 0 & 0 & 1032 \\
1997 & 653 & 0 & 0 & 653 \\
\hline 1998 & 790 & 1 & 0 & 791 \\
\hline 1999 & 1884 & 1 & 0 & 1885 \\
\hline 2000 & 1308 & 756 & 1 & 2065 \\
\hline 2001 & 918 & 721 & 0 & 1639 \\
\hline 2002 & 5418 & 1004 & 2 & 6424 \\
2003 & 1396 & 760 & 8 & 2164 \\
\hline 2004 & 1283 & 1042 & 2 & 2327 \\
\hline 2005 & 1080 & 1534 & 4 & 2618 \\
\hline 2006 & 990 & 1154 & 1 & 2145 \\
\hline 2007 & 772 & 976 & 1 & 1749 \\
\hline 2008 & 706 & 856 & & 1562 \\
2009 & 512 & 901 & 1 & 1414 \\
\hline Total & 19006 & 9706 & 20 & 28732 \\
\hline
\end{tabular}

Se observa que en el periodo estudiado, los indicadores de investigación oportuna en 48 horas después de la notificación y la investigación adecuada han permanecido por debajo del $80 \%$ (rango 1); el indicador de notificación semanal en el periodo de 1996-2007 se mantuvo por encima del $80 \%$ (rango 2), en los años 2008 y 2009 presentó el $74 \%$ y $71 \%$ respectivamente. En cuanto al indicador de toma de muestra adecuada para el período 1990-2009 reportó en promedio $93,7 \%$, para la recepción de muestra -menor o igual a 5 días-, para el periodo de 1995-2006 el promedio de cumplimiento fue de 65,2 $\%$ y para 2007-2009 el promedio de cumplimiento para este indicador fue de $83,3 \%$. Para el indicador de resultados de laboratorio -menor o igual a 4 díasentre 1995-2000 el promedio de cumplimiento fue de 55\% y entre 2001-2009 de $86,8 \%$ (Tabla 4 ).

La tasa de notificación de casos sospechosos de sarampión y rubéola en los años 1995 y 1997 fue de 0,70 y 1,69 casos por 100000 habitantes respectivamente. Para los otros años del periodo en estudio se presentó una tasa por encima de 2 casos por 100000 habitantes (Tabla 5).La mayor incidencia por sarampión reportada fue para el 2002 con 0,33 casos por 100000 habitantes, en el periodo de 2003-2009 fue de 0 casos por 100000 habitantes. Para rubéola la mayor incidencia se reportó en 1996 con 0,57 casos por 100000 habitantes y la menor para el año 2009 con 0.0089 casos por 100000 habitantes (Tabla 6). 


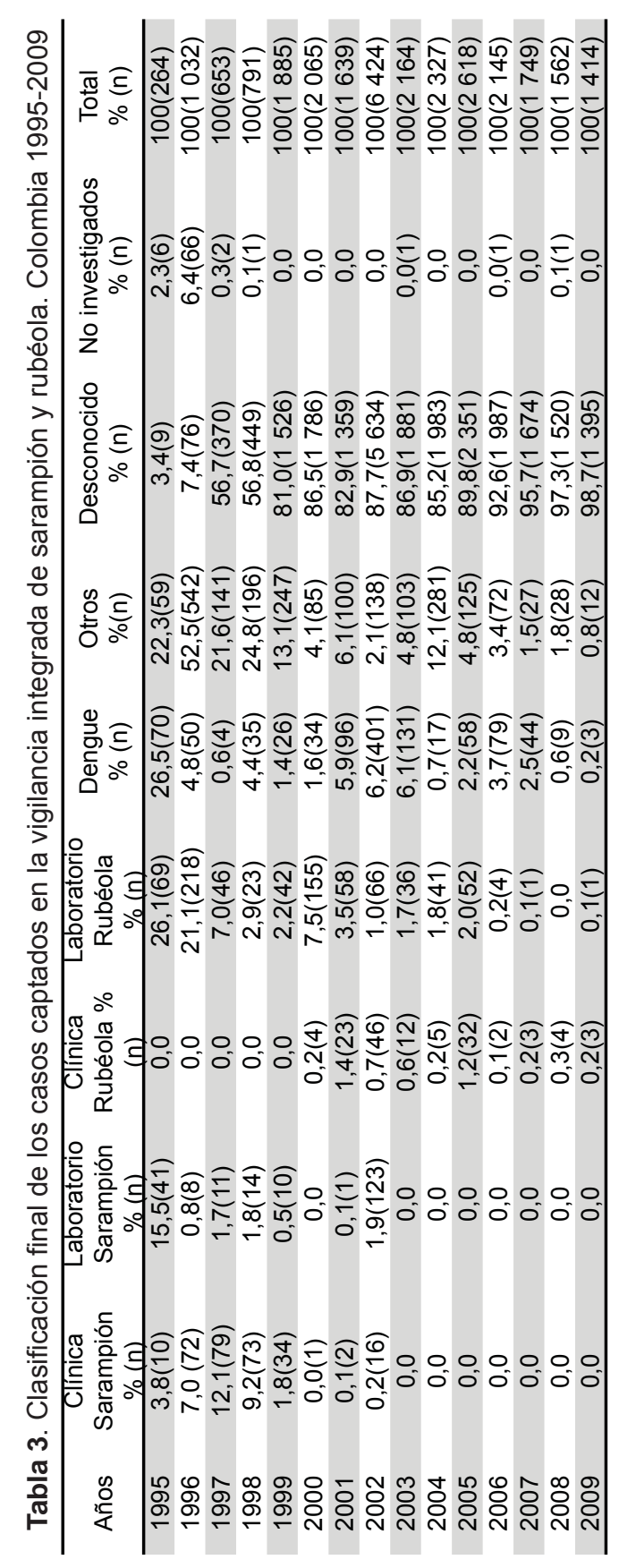


Tabla 4. Indicadores de calidad de la vigilancia integrada de sarampión y rubéola. Colombia 1995-2009

\begin{tabular}{ccccccc}
\hline Año & $\begin{array}{c}\text { Unidades } \\
\text { que notifican } \\
\text { semanal } \%\end{array}$ & $\begin{array}{c}\text { Casos } \\
\text { investigados } \\
48 \text { horas } \%\end{array}$ & $\begin{array}{c}\text { Investigación } \\
\text { adecuada } \%\end{array}$ & $\begin{array}{c}\text { Muestras } \\
\text { adecuadas } \\
\%\end{array}$ & $\begin{array}{c}\text { Muestras } \\
\text { recibidas } \\
\leq 5 \text { días } \%\end{array}$ & $\begin{array}{c}\text { Resultados } \\
\text { laboratorios } \\
\leq 4 \text { días \% }\end{array}$ \\
\hline 1995 & 38 & 38 & 8 & 31 & 33 & 11 \\
1996 & 84 & 56 & 14 & 44 & 57 & 66 \\
1997 & 97 & 61 & 28 & 82 & 52 & 55 \\
1998 & 90 & 58 & 25 & 69 & 55 & 62 \\
1999 & 87 & 81 & 43 & 90 & 64 & 64 \\
2000 & 87 & 67 & 42 & 97 & 74 & 72 \\
2001 & 83 & 55 & 33 & 95 & 69 & 81 \\
2002 & 84 & 42 & 22 & 88 & 76 & 84 \\
2003 & 88 & 58 & 32 & 94 & 70 & 82 \\
2004 & 92 & 58 & 35 & 96 & 76 & 90 \\
2005 & 93 & 61 & 36 & 93 & 78 & 89 \\
2006 & 92 & 66 & 45 & 96 & 79 & 84 \\
2007 & 88 & 68 & 49 & 97 & 80 & 90 \\
2008 & 74 & 68 & 42 & 92 & 85 & 92 \\
2009 & 71 & 70 & 50 & 93 & 85 & 90 \\
\hline
\end{tabular}

Tabla 5. Tasa de notificación de casos sospechosos de sarampión y rubéola. Colombia 1995-2009

\begin{tabular}{cccc}
\hline Año & $\begin{array}{c}\text { Sospechosos } \\
\text { sarampión/rubéola }\end{array}$ & $\begin{array}{c}\text { Población } \\
\text { total }\end{array}$ & $\begin{array}{c}\text { Tasa notificación } \\
\text { por } 100 \text { 000 habitantes }\end{array}$ \\
\hline 1995 & 264 & 37489666 & 0,70 \\
1996 & 1032 & 38076638 & 2,71 \\
1997 & 653 & 38646043 & 1,69 \\
1998 & 791 & 39201321 & 2,02 \\
1999 & 1885 & 39745714 & 4,74 \\
2000 & 2065 & 40282217 & 5,13 \\
2001 & 1639 & 40806313 & 4,02 \\
2002 & 6424 & 41327459 & 15,54 \\
2003 & 2164 & 41847421 & 5,17 \\
2004 & 2327 & 42367528 & 5,49 \\
2005 & 2618 & 42888592 & 6,10 \\
2006 & 2145 & 43405387 & 4,94 \\
2007 & 1749 & 43926034 & 3,98 \\
2008 & 1562 & 44450260 & 3,51 \\
2009 & 1414 & 44977758 & 3,14 \\
\hline
\end{tabular}


Tabla 6. Incidencia de sarampión y rubéola por 100000 habitantes.

\begin{tabular}{cccccc}
\multicolumn{5}{c}{ Colombia1995-2009 } \\
\hline Año & Población & $\begin{array}{c}\text { Confirmados } \\
\text { sarampión }\end{array}$ & $\begin{array}{c}\text { Confirmados } \\
\text { rubéola }\end{array}$ & $\begin{array}{c}\text { Incidencia } \\
\text { sarampión por } \\
100 \text { 000 Hab. }\end{array}$ & $\begin{array}{c}\text { Incidencia } \\
\text { rubéola por } \\
100 \text { 000 Hab. }\end{array}$ \\
\hline 1995 & 37489666 & 51 & 69 & 0,1360 & 0,1841 \\
1996 & 38076638 & 80 & 218 & 0,2101 & 0,5725 \\
1997 & 38646043 & 90 & 46 & 0,2329 & 0,1190 \\
\hline 1998 & 39201321 & 87 & 23 & 0,2219 & 0,0587 \\
1999 & 39745714 & 44 & 42 & 0,1107 & 0,1057 \\
2000 & 40282217 & 1 & 159 & 0,0025 & 0,3947 \\
2001 & 40806313 & 3 & 81 & 0,0074 & 0,1985 \\
2002 & 41327459 & 139 & 112 & 0,3363 & 0,2710 \\
2003 & 41847421 & 0 & 48 & 0,0000 & 0,1147 \\
2004 & 42367528 & 0 & 46 & 0,0000 & 0,1086 \\
2005 & 42888592 & 0 & 84 & 0,0000 & 0,1959 \\
2006 & 43405387 & 0 & 6 & 0,0000 & 0,0138 \\
2007 & 43926034 & 0 & 4 & 0,0000 & 0,0091 \\
2008 & 44450260 & 0 & 4 & 0,0000 & 0,0090 \\
2009 & 44977758 & 0 & 4 & 0,0000 & 0,0089 \\
\hline
\end{tabular}

\section{DISCUSIÓN}

Las estrategias implementadas por Colombia en estos años, a partir de la incorporación de la vacuna en forma programática y en campañas, sumado a la implementación de un sistema de vigilancia activa, han contribuido al éxito de la interrupción de la transmisión del sarampión y a avanzar rápidamente hacia la eliminación de la rubéola y síndrome de rubéola congénita.

En éste trabajo se describe la vigilancia epidemiológica del sarampión y la rubéola, y se evidencia una vigilancia activa entre 1995 y 2009 ; adicionalmente, demuestra en general para el periodo en estudio una alta sensibilidad (tasa de notificación), cuyo umbral mínimo es de $\geq 2$ casos por 100000 habitantes definido internacionalmente (8); permite adicionalmente observar el progreso logrado en la eliminación (22). Por otro lado, a través de la información disponible se ratifica que después del 2002 no se han presentado más casos confirmados de sarampión en el país, para rubéola el último caso confirmado reportado es del año 2009. Los indicadores del laboratorio que evalúan la calidad de respuesta ante casos sospechosos de sarampión y rubéola han demostrado una continua y conservada mejoría, demostrado con indicadores por encima de la meta establecida (21). Los indicadores de unidades que notifican semanalmente, investigación en 48 horas e investigación adecuada, han demostrado un inadecuado desempeño sin conseguir la meta establecida, por tanto es necesario fortalecer estos procesos para aumentar la respuesta 
inmediata ante casos sospechosos de estos eventos. En los dos últimos años del periodo estudiado (2008-2009), el país cumplió con 4 de los 7 indicadores que evalúan la calidad de la vigilancia integrada del sarampión y la rubéola.

La vigilancia integrada de sarampión-rubéola (23) es una estrategia importante que ha demostrado un papel primordial en el mantenimiento de la eliminación de estos eventos en Colombia; la eliminación de estas enfermedades no equivale a una incidencia igual a cero sino conseguir o mantener en cero la transmisión secundaria teniendo en cuenta el riesgo de importación constante. Una vigilancia sensible es indispensable para la eliminación del sarampión y rubéola; abarca la notificación y la investigación oportuna de los casos sospechosos. Las enfermedades sujetas a programas de eliminación, requieren de mecanismos que permitan una evaluación permanente de la sensibilidad del sistema de vigilancia y asegurar la captación de la totalidad de los casos sospechosos con la investigación con calidad de cada uno de ellos (24).

El plan de eliminación del sarampión y la rubéola en Colombia ha sido una tarea de gran alcance que ha requerido el trabajo conjunto de los actores que integran el sistema de vigilancia y control en salud pública, como también la colaboración de las organizaciones internacionales y multilaterales. En el país durante el transcurso de los años se han adoptado nuevos enfoques de vacunación además de la vigilancia epidemiológica y virológica de forma rutinaria que ha logrado un impacto positivo en la incidencia del sarampión, la rubéola y el síndrome de rubéola congénita

Conflicto de intereses: Ninguno

\section{REFERENCIAS}

1. Instituto de Salud Carlos III. Centro Nacional de Epidemiología. Área de Vigilancia de la Salud Pública. Red Nacional de Vigilancia Epidemiológica de España. Informe Anual del Plan de Eliminación del Sarampión, Rubéola y Síndrome de Rubéola Congénita en España; 2000. p.4.

2. Organización Mundial de la Salud. Sarampión [Internet]. Disponible en: http://www.who.int/ mediacentre/factsheets/fs286/es/index.html. Consultado enero de 2011.

3. Organización Mundial de la Salud. ¿Qué dimensiones tiene el problema del sarampión? [Internet]. Disponible en: http://www.who.int/mediacentre/factsheets/fs286/es/index. htm.Consultado enero de 2011.

4. Organización Mundial de la Salud. Measles Initiative [Internet]. Disponible en: http://www. measlesinitiative.org/. Consultado enero de 2011. 
5. Organización Mundial de la Salud. Consejo Ejecutivo. 125a reunión [Internet]. Punto 5.1 del orden del día provisional. Eliminación Mundial del Sarampión. EB 125/4 16 de abril de 2009.]. Disponible en: http://apps.who.int/gb/ebwha/pdf_files/EB125/B125_4sp.pdf Consultado enero de 2011.

6. Organización Mundial de la Salud. $63^{\mathrm{a}}$ Asamblea Mundial de la Salud. Erradicación mundial del sarampión. Informe de la Secretaría. A63/18; 26 de marzo de 2010. p. 1-8.

7. Organización Panamericana de la Salud. Organización Mundial de la Salud. $44^{\circ}$ Consejo Directivo. 55 Sesión del Comité Regional. Resolución CD44.R1. Mantenimiento de los Programas de Vacunación-Eliminación de la rubéola y el síndrome de rubéola congénita (SRC); septiembre de 2003. p. 1-3.

8. Organización Panamericana de la Salud [Internet]. Área de Salud Familiar y Comunitaria. Proyecto de Inmunización Integral de la Familia. Plan de acción para la documentación y verificación de la eliminación de sarampión, rubéola y síndrome de rubéola congénita en la región de las Américas. Documento técnico, Noviembre de 2009. Disponible en: http://www.bvsde.paho.org/texcom/sct/048018.pdf. Consultado marzo de 2011.

9. Organización Panamericana de la Salud. Organización Mundial de la Salud. 140a Sesión del Comité Ejecutivo. Eliminación de la rubéola y del síndrome de rubéola congénita en las Américas: Informe sobre los progresos realizados. Punto 4.3 del orden del día provisional CE140/8 (Esp.). Mayo 2007. Washington, D.C., EUA; junio de 2007; p. 1-8.

10. Rodríguez MÁ, Restrepo C, Uribe G. Estado serológico para sarampión en población de 1 a 14 años, Medellín 1996. Colombia Médica. Universidad del Valle. 1999; 30 (2):82-8.

11. Velandia $M$, Castillo $O$, Higuera $A B$, Pastor D. Eliminación de la rubéola y el síndrome de rubéola congénita, Plan de acción 2004-2010. Inf Quinc Epidemiol Nac. 2004; 9(21):321-36.

12. Castillo $O$, Pastor $D$ [Internet]. Evaluación del plan de Erradicación del sarampión en Colombia 1999 - SIVIGILA No. 06 Febrero 6 al 12 de 2000.].Disponible en: http:// www.col.ops-oms.org/pai/plansarampion.htm.Consultado enero de 2011.

13. Organización Panamericana de la Salud. Oficina Sanitaria Panamericana. Oficina Regional de la Organización Mundial de la Salud. Reuniones subregionales Andina y del Cono Sur sobre Enfermedades Prevenibles por Vacunación, 1 al 3 de septiembre de 2003. Boletín Informativo PAI. Programa Ampliado de Inmunización en las Américas. 2003; (5):2-4.

14. Organización Mundial de la Salud [Internet]. Conclusiones de la $63^{\mathrm{a}}$ Asamblea Mundial de la Salud con varias resoluciones adoptadas Erradicación mundial del sarampión.]. Disponible en: http://www.who.int/mediacentre/news/releases/201)0/wha_ closes_20100521/es/index.html. Consultado enero de 2011.

15. Programa de las Naciones Unidas para el Desarrollo. PNUD [Internet].Objetivos de desarrollo del Milenio ODM. Disponible en: http://www.undp.org/spanish/mdg/ basics.shtml. Consultado febrero 2011. 
16. Castillo O, Rey G, Velandia M, Pastor D. Situación del sarampión en Colombia, 2002. Inf Quinc Epidemiol Nac. 2003;8(8):129-44.

17. República de Colombia. Ministerio de la Protección Social. Decreto por el cual se crea y reglamenta el Sistema de Vigilancia en Salud Pública y se dictan otras disposiciones. Decreto 3518 de 2006; (09 de octubre).

18. República de Colombia. Ministerio de la Protección Social. Manual Técnico Administrativo del Programa Ampliado de Inmunizaciones; 2008.

19. Organización Panamericana de la Salud. Unidad de Inmunización Salud Familiar y Comunitaria. Curso de gerencia para el manejo efectivo del Programa Ampliado de Inmunización (PAI). Módulo IV Vigilancia epidemiológica; 2006.

20. Londoño JL. Metodología de la investigación epidemiológica, $3^{a}$ Edición, Bogotá, Editorial El Manual Moderno; 2004.

21. Hernández M. Epidemiología. Diseño y Análisis de Estudios. Instituto Nacional de Salud Pública. México: Editorial Médica Panamericana, SA de C.V.; 2007.

22. Organización Panamericana de la Salud. Eliminación del sarampión, guía práctica, segunda edición. Washington; 2007.

23. Gallegos D. Sarampión y Rubéola. Enfermedades en vías de eliminación en América. Enfermedades de Notificación Obligatoria. Boletín de Vigilancia en Salud Pública en Chile. El Vigía. 2003; 19: 33-36.

24. Instituto Nacional de Salud [Internet].Protocolo Vigilancia integrada sarampión y rubéola. Disponible en: http://www.ins.gov.co/?idcategoria=50184. Consultado febrero de 2011. 